\title{
Arthroscopic Fixation of Cell Free Polymer-Based Cartilage Implants with a Bioinspired Polymer Surface on the Hip Joint: A Cadaveric Pilot Study
}

\author{
Matthias Lahner, ${ }^{1}$ Christian Duif, ${ }^{1}$ Andreas Ficklscherer, ${ }^{2}$ Christian Kaps, ${ }^{3,4}$ \\ Lukas Kalwa, ${ }^{5}$ and Tobias Seidl ${ }^{5}$ \\ ${ }^{1}$ Department of Orthopaedic Sports Surgery, Ruhr-University Bochum, St. Josef-Hospital, Gudrunstrße 56, 44791 Bochum, Germany \\ ${ }^{2}$ Department of Orthopaedic Surgery, Physical Medicine and Rehabilitation, University Hospital of Munich (LMU), \\ Campus Großhadern, Marchioninistraße 15, 81377 München, Germany \\ ${ }^{3}$ BioTissue Technologies GmbH, Charitéplatz 1, 10117 Berlin, Germany \\ ${ }^{4}$ Tissue Engineering Laboratory, Department of Rheumatology and Clinical Immunology, Charité-Universitätsmedizin Berlin, \\ Charitéplatz 1, 10117 Berlin, Germany \\ ${ }^{5}$ Westphalian Institute for Biomimetics, Westphalian University of Applied Sciences, Münsterstraße 265, 46397 Bocholt, Germany
}

Correspondence should be addressed to Matthias Lahner; m.lahner@klinikum-bochum.de

Received 18 April 2014; Accepted 5 August 2014; Published 28 August 2014

Academic Editor: Martin Stoddart

Copyright (C) 2014 Matthias Lahner et al. This is an open access article distributed under the Creative Commons Attribution License, which permits unrestricted use, distribution, and reproduction in any medium, provided the original work is properly cited.

\begin{abstract}
This study investigates the adhesion capacity of a polyglycolic acid- (PGA-) hyaluronan scaffold with a structural modification based on a planar polymer (PM) surface in a cadaver cartilage defect model. Two cadaver specimens were used to serially test multiple chondral matrices. In a cadaver hip model, cell free polymer-based cartilage implants with a planar bioinspired PM surface (PGA-PM-scaffolds) were implanted arthroscopically on $10 \mathrm{~mm} \times 15 \mathrm{~mm}$ full-thickness femoral hip cartilage lesions. Unprocessed cartilage implants without a bioinspired PM surface were used as control group. The cartilage implants were fixed without and with the use of fibrin glue on femoral hip cartilage defects. After 50 movement cycles and removal of the distraction, a rearthroscopy was performed to assess the outline attachment and integrity of the scaffold. The fixation techniques without and with fibrin fixation showed marginal differences for outline attachment, area coverage, scaffold integrity, and endpoint fixation after 50 cycles. The PGA-PM-scaffolds with fibrin fixation achieved a higher score in terms of the attachment, integrity, and endpoint fixation than the PGA-scaffold on the cartilage defect. Relating to the outline attachment, area coverage, scaffold integrity, and endpoint fixation, the fixation with PGA-PM-scaffolds accomplished significantly better results compared to the PGA-scaffolds $(P=0.03752, P=$ $0.03078, P=0.00512, P=0.00512)$. PGA-PM-scaffolds demonstrate increased observed initial fixation strength in cadaver femoral head defects relative to PGA-scaffold, particularly when fibrin glue is used for fixation.
\end{abstract}

\section{Introduction}

In the regenerative cartilage surgery, many surgical techniques were developed for coating focal articular cartilage defects. Current available surgical cartilage treatments include lavage and debridement, subchondral penetration procedures (microfracture technique, drilling, or abrasion chondroplasty), autogenous osteochondral transplantation (AOT), and autologous chondrocyte implantation (ACI) with or without using a scaffold [1-7].
As a single-stage procedure, the most used treatment of focal cartilage defects represents the microfracture (Mfx) technique in which a penetration of the subchondral bone layer is performed with subsequent effluent of progenitor cells from the bone marrow into the articular cartilage lesion $[1,2,8]$. The progenitor cells are similar to chondrocytes but form a fibrocartilaginous tissue which is mechanically and biochemically substandard to the originary cartilage tissue [9]. 
Scaffold-assisted single-step techniques were developed to enhance cartilage tissue regeneration by the combination of the Mfx with a biomaterial. By combination of the Mfx with a scaffold, the mesenchymal stem cells (MSCs) are attracted into the biomaterial and the chondrogenic differentiation of the MSCs to cartilage repair tissue is better assured such as the sole application of the Mfx $[10,11]$. However, the fibrin clot is not mechanically stable, so the implanted scaffold enhances the mechanical stability in the defect zone additionally [12]. The biomaterial serves as a biostructure for cell attachment of the MSCs [13]. Different matrices are currently available for surgical cartilage repair like scaffolds with porcine collagen I/III membrane [14, 15]. Another scaffolding for cartilage repair is a cell free matrix composed of an absorbable nonwoven polyglycolic acid (PGA) textile combined with hyaluronic acid (HA) $[16,17]$. The mechanical stability of the matrix allows for easy treatment and safe fixation in the cartilage lesion by fibrin sealant, cartilage, or transosseous suture, or by resorbable pins [18, 19]. However, the arthroscopic pin insertion for fixation of the scaffold represents a challenge during arthroscopic cartilage repair. An incorrect placed pin can release cartilage damage of the articular opposite side [20].

The importance of the primary fixation of the scaffolds is common, but there is no study available which examines the influence of a structural modification of the scaffold surface in order to achieve a higher adhesion ability [21].

Here we compare and quantify the primary fixation stability of a commonly used scaffold for cartilage repair attached by a planar bioinspired polymer (PM) surface in a cadaver hip model. The aim of our experimental study was that the arthroscopic implantation of a cell free polymerbased scaffold with a structure derived from tree-frog footpads was more stabile compared to an unprocessed scaffold.

\section{Materials and Methods}

2.1. Testing Protocol. The study was approved by the local ethical committee of the Ruhr-University Bochum (registration number 4611-13). The surgery was performed by two orthopaedic surgeons experienced in hip arthroscopy (Matthias Lahner, Christian Duif). The fluoroscope was placed diagonally across the table (Philips, Hamburg, Germany). In this study, two right human cadaver hips were arranged for hip arthroscopy in a supine position demonstrated in Figure 1. Arthroscopy for the central joint compartment was performed with distraction. The ventral portal was created under fluoroscope. The hip capsule was widened through a $1.5 \mathrm{~mm}$ guide wire with sequential telescoping $4 \mathrm{~mm}$ and $6 \mathrm{~mm}$ portal dilators (Arthrex, Naples, FL, USA). After advancing a $5.5 \mathrm{~mm}$ fenestrated metal cannula into the joint, an arthroscopic evaluation was performed with a $70^{\circ} 4 \mathrm{~mm}$ scope (Arthrex). A second instrumentation portal approach was established with a 14-gauge needle under arthroscopic and fluoroscopic view. The hip capsule was penetrated with a $4 \mathrm{~mm}$ dilator and an $8 \mathrm{~mm} \times 4 \mathrm{~cm}$ pass port button cannula (Arthrex) which was positioned to create a stable portal. By using a bone cutter (Arthrex), a $10 \times 15 \mathrm{~mm}$

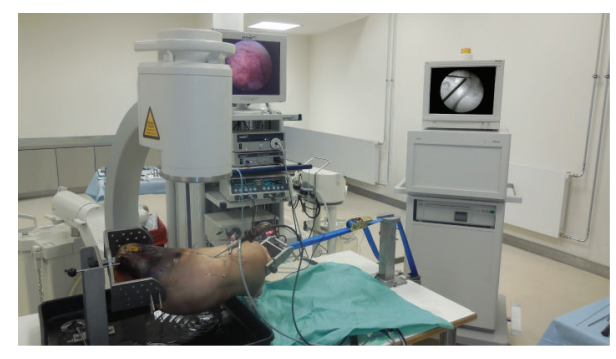

FIgURE 1: Experimental setup with arthroscopy and fluoroscopy equipment.

full-thickness cartilage defect was created in the zone 2 of the femoral head (hip 1 and 2) described by Ilizaliturri et al. [22]. Then different scaffolds were placed into the defect, either a PGA-hyaluronan scaffold without structural modification (Figure 2) or a bioinspired PGA-PM-scaffold (Figure 3). Both groups were implanted with or without fibrin glue (Evicel, Omrix Biopharmaceuticals NV, Diegem, Belgium). Both cadavers had all four treatments.

2.2. Testing Groups. The resorbable PGA-hyaluronan scaffold (BioTissue Technologies GmbH, Freiburg, Germany) of $10 \mathrm{~mm} \times 15 \mathrm{~mm} \times 1.1 \mathrm{~mm}$ was impregnated with an embossing stamp. The embossing stamp consisted of a thermoplastic polymer (PM) surface composed of acrylnitrile butadiene styrene (ABS). The PM was designed of a moulding form with computer-aided design (CAD) software from a 3D printer (Dimension BST, Stratasys, Eden Prairie, USA). The PGAhyaluronan scaffold was loaded with the PM embossing stamp with a defined 4 kilo force for about 20 minutes in a laboratory-type drying cabinet at $80^{\circ} \mathrm{C}$. The PGA-PMscaffold was produced only for experimental use. For the fixation, the scaffold was placed directly onto the defect of the subchondral bone without additional material.

2.3. Arthroscopic Implantation. After evacuating the saline solution, the scaffold was implanted by the use of a tissue grasper and simply released above the full-thickness cartilage defect. By using a tissue elevator, the scaffolds were shaped into the defect to reach a $100 \%$ filling of the defect. Before fixation of the scaffold with fibrin glue, we used an application device with a $35 \mathrm{~cm}$ tip (Evicel) through the instrumentation portal. After removing the distraction, 50 cycles of flexion $\left(90^{\circ}\right)$ and extension $\left(10^{\circ}\right)$ were performed by moving the leg manually. The cycles were conducted by an independent third-party-assistant that was blinded to the assessment of the measurements. Then, the hip joint was refilled with saline solution and the defect area with the implanted scaffold was inspected (Figures 4 and 5). After the implantation of scaffold, the matrix was completely removed and the fibrin glue was eliminated by the shaver. All hip cadavers underwent the presented motion protocol with two fixation techniques tested on each hip. The outline attachment and the percentage of defect-covering scaffold were noticed as well as the integrity of the scaffold itself. The endpoint fixation strength was manually tested by a palpating hook. 


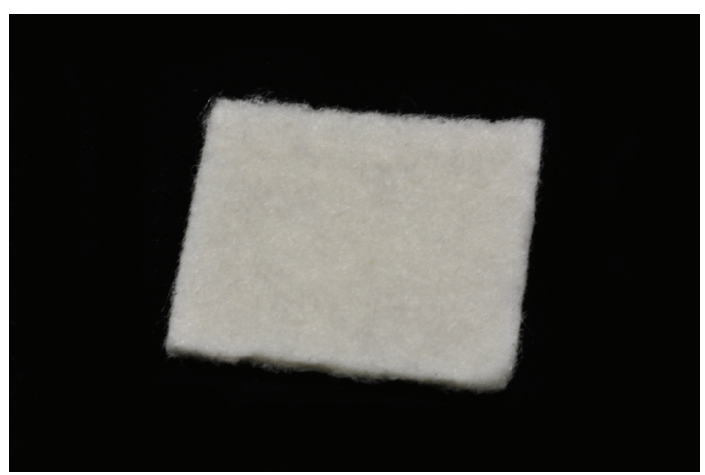

(a)

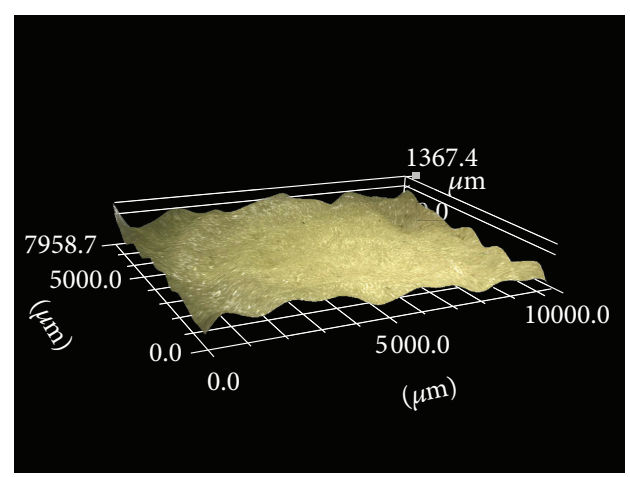

(b)

FIGURE 2: Polyglycolic acid- (PGA-) hyaluronan scaffold (a) and confocal stereomicroscopic picture of the scaffold before fixation (b).

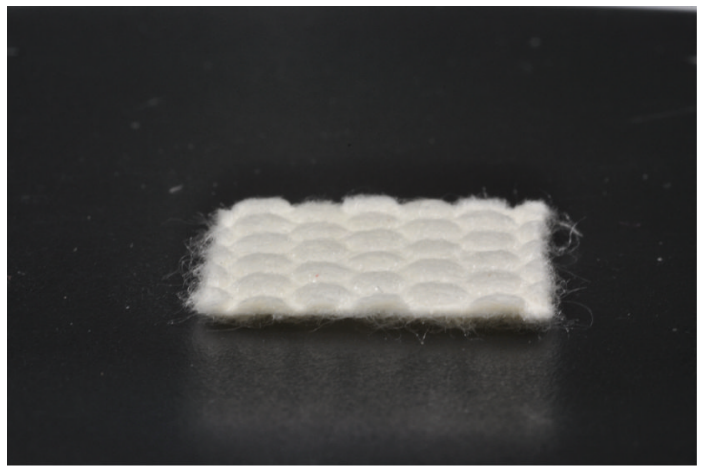

(a)

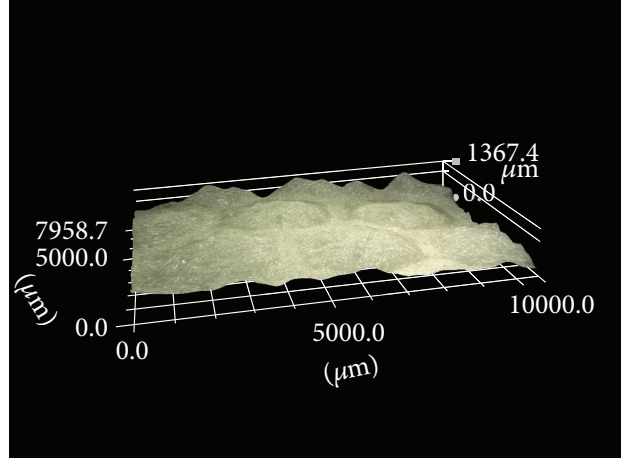

(b)

FIGURE 3: PGA-scaffold with polymer (PM) surface (a) and confocal stereomicroscopic picture of the scaffold before fixation (b).

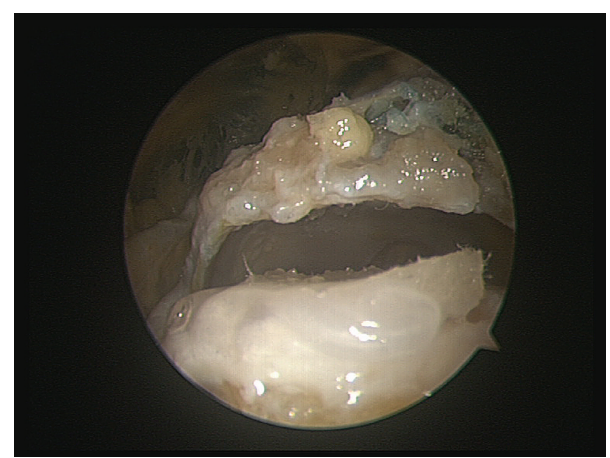

Figure 4: Implantation of the PGA-scaffold on a full-thickness femoral hip cartilage defect. The implant was used to cover the defect and fixed by a fibrin glue.

The properties of the scaffold were directly evaluated by two orthopedic surgeons. All scaffolds were photographed at each rearthroscopy and reviewed by the third collaborator who did not participate in the initial surgery. We performed therefore three measurements per fixation. A consensus of all three evaluators was taken as the end result.

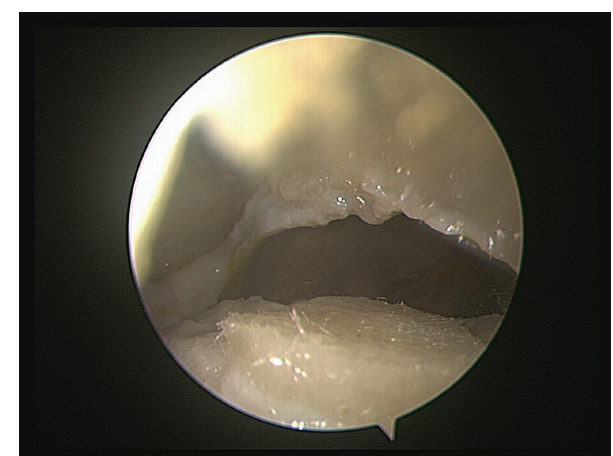

FIGURE 5: Implantation of the PGA-scaffold modified with a planar PM surface. The implant was used to cover the defect and fixed by a fibrin glue.

2.4. Classification of Scaffold Evaluation. To classify the differences concerning adhesion and integrity between the tested scaffolds, we modified the scoring system developed by Bekkers et al. [23]. Outline attachment, area coverage, scaffold integrity, and endpoint fixation were assessed and a corresponding score was determined in a 5-point scale (Table 1). 
TABLE 1: Modified criteria for the scaffold evaluation described by Bekkers et al. [23]. The assigned points are given in brackets.

\begin{tabular}{llll}
\hline $\begin{array}{l}\text { Outline } \\
\text { attachment }\end{array}$ & Area coverage & Scaffold integrity ${ }^{* *}$ & Endpoint fixation ${ }^{* * *}$ \\
\hline Unchanged (5) & Unchanged (5) & Unchanged (5) & Cannot be detached (5) \\
\hline$<25 \%(4)$ & $\begin{array}{l}\text { Shape deformities without structural } \\
\text { damage (4) }\end{array}$ & $\begin{array}{l}\text { Shape deformities or minor fissures that } \\
\text { are unrelated to fixation (4) }\end{array}$ & Detached with intensive pull (4) \\
\hline $25-50 \%(3)$ & $\begin{array}{l}\text { Fissures or cracks without important } \\
\text { substances loss (3) }\end{array}$ & $\begin{array}{l}\text { Minor fissures or cracks close to the } \\
\text { fixation site (3) }\end{array}$ & Detached with minor pull (3) \\
\hline $50-75 \%(2)$ & $<25 \%$ of scaffold lost (2) & $\begin{array}{l}\text { Fissures or cracks endangering the } \\
\text { fixation of the scaffold (2) }\end{array}$ & Detached with slight touch (2) \\
\hline $75-100 \%(1)$ & $25-50 \%$ of scaffold lost (1) & $\begin{array}{l}\text { Fissures or cracks endangering the } \\
\text { fixation with surrounding scaffold } \\
\text { disorganization (1) }\end{array}$ & Partial detachment (1) \\
\hline $100 \%(0)$ & $>50 \%$ of scaffold lost (0) & $\begin{array}{l}\text { Fissures or cracks endangering the } \\
\text { fixation with generalized scaffold } \\
\text { disorganization }(0)\end{array}$ & Total detachment (0) \\
\hline
\end{tabular}

* $\%$ of full circumference that has lost contact with the surrounding cartilage rim.

** $\%$ of total cartilage defect that is covered by scaffold.

*** The endpoint fixation was tested by a palpation hock manipulation after the motion cycles were completed.

TABLE 2: Results of two scaffold fixation techniques on two human cadavers after 50 cycles of continuous motion.

\begin{tabular}{lccccc}
\hline Material & $\begin{array}{c}\text { Fixation } \\
\text { technique }\end{array}$ & $\begin{array}{c}\text { Outline attachment } \\
50 \text { cycles average score } \\
( \pm \text { standard deviation })\end{array}$ & $\begin{array}{c}\text { Area coverage } \\
50 \text { cycles average score } \\
( \pm \text { standard deviation })\end{array}$ & $\begin{array}{c}\text { Scaffold integrity } \\
50 \text { cycles average score } \\
( \pm \text { standard deviation })\end{array}$ & $\begin{array}{c}\text { Endpoint fixation } \\
50 \text { cycles average score } \\
( \pm \text { standard deviation })\end{array}$ \\
\hline PGA-scaffold & Unfixed & $1.0( \pm 0)$ & $1.5( \pm 0.7)$ & $1.0( \pm 0)$ & $0.5( \pm 0.7)$ \\
\hline PGA-PM-scaffold & Unfixed & $2.0( \pm 0)^{*}$ & $2.0( \pm 0)$ & $3.0( \pm 0)$ & $2.0( \pm 0)^{*}$ \\
\hline PGA-scaffold & $\begin{array}{l}\text { Fixed with } \\
\text { fibrin glue }\end{array}$ & $2.5( \pm 0.7)$ & $2.5( \pm 0.7)$ & $4.0( \pm 0.7)^{*}$ & $3.0( \pm 0.0)$ \\
\hline PGA-PM-scaffold & $\begin{array}{l}\text { Fixed with } \\
\text { fibrin glue }\end{array}$ & $3.5( \pm 0.7)^{*}$ & $3.5( \pm 0.7)^{*}$ & $3.0)^{*}$ \\
\hline
\end{tabular}

Average per scoring item for the scaffold fixation techniques after 50 cycles $\left({ }^{*} P<0.05\right)$.

2.5. Statistical Analysis. For each individual fixation technique, the average scores and standard deviations per scoring item were calculated after 50 motion cycles. The arithmetic mean value and the SD were calculated for the variables above and measured with Microsoft Excel (Microsoft, Redmond, WA, USA). The values were recorded in IBM SPSS Statistics 22 (PASW 22, SPSS Inc., Chicago, IL, USA). Mean values of each set were performed using Mann-Whitney $U$ test with level of significance defined at ${ }^{*} P<0.05$.

\section{Results}

All the fixation techniques were feasible through the initial incision. None of the specimens showed macroscopic damage at the opposing articular cartilage surface. We compared the unfixed PGA-scaffold versus the unfixed PGA-PM-scaffold plus the PGA-scaffold with fibrin glue versus the PGA-PMscaffold with fibrin glue. The results of the scaffold fixation are shown in Table 2. Concerning the scaffolds fixed with fibrin glue, the fixation with PGA-PM-scaffolds accomplished significantly better results compared to the PGA-scaffolds relating to the outline attachment, area coverage, scaffold integrity, and endpoint fixation $(P=0.03752, P=0.03078$, $P=0.00512, P=0.00512)$. Concerning the outline attachment and endpoint fixation, lower statistically significant scores were found for the unfixed PGA-PM-scaffold compared to the PGA-scaffold ( $P=0.02034, P=0.01314)$. With regard to the area coverage and scaffold integrity, no statistical difference was observed between the unfixed PGAscaffold and the unfixed PGA-PM-scaffold after 50 cycles $(P=0.17384 P=0.06576)$. Closer examination showed an incomplete detachment of the PGA-scaffold without fibrin glue to a complete attachment of the PGA-PM-scaffold with adhesive fixation. However, no rupture of the scaffold fiber was observed during arthroscopic implantation. Relating to the scaffold integrity, the PGA-PM-scaffold with fibrin glue achieved the best result after the motion cycles. The fibrin glue fixation with PGA-PM-scaffolds provided the best scaffold integrity as compared to scaffolds without adhesive fixation after 50 cycles. The endpoint fixation strength of the scaffold was highest in the PGA-PM-scaffold fixed with fibrin glue. Table 3 shows a summary concerning the advantages and disadvantages as well as the pros and cons of the clinical procedure of the PGA-PM-scaffolds.

\section{Discussion}

In the present study, we assessed the primary arthroscopic fixation stability of scaffold-based tissue-engineered implants 
TABLE 3: Summary of the clinical procedure of the PGA-PM-scaffolds.

\begin{tabular}{llll}
\hline Advantages & Disadvantages & Pros & Cons \\
\hline Single-stage procedure & Demanding arthroscopic technique & Minimal invasiveness & Unsuitable for larger \\
Suitable for medium chondral defects $\left(2-4 \mathrm{~cm}^{2}\right)$ & $\begin{array}{l}\text { Demanding production of the } \\
\text { implant }\end{array}$ & $\begin{array}{l}\text { procedure } \\
\text { Rapid rehabilitation }\end{array}$ & $\begin{array}{l}\left(>4 \mathrm{~cm}^{2}\right) \\
\text { Low operative cost }\end{array}$
\end{tabular}

of full-thickness cartilage defects in cadaver hips to simulate the adhesion properties. Regarding the fixation, we showed that the adherence on the cartilage defect was higher with the PGA-PM-scaffold than with the unmodified PGA-scaffold. These results were collected in experimental settings with high similarity to the intended application. They confirm the initial assessment from the development process.

This study was limited by the reduced number of implanted scaffolds and the lack of power analysis. Another limitation of our study was that we did not apply static uniaxial tensile tests on in situ cadaver legs; thus the transfer of the findings to a clinical setting is difficult. However, we did not accomplish intra-articular pressure measuring, but it can be assumed that the anatomical precondition of the hip joint with additional burden during motion generated a shearing force on the scaffold, which is similar to the in vivo conditions. This shear force will partially be conveyed directly by friction with the opposing cartilage and partially by the intra-articular remaining saline solution. Further biomechanical studies are needed to prove whether a slightly submerged implant could protect it from the friction in vivo, although in one rabbit model trial, it was demonstrated that submerged metal grafts in focal cartilage lesion deranged the integrity of the articulating cartilage surfaces [24].

Bekkers et al. demonstrated the need for a compromise between fixation stability and scaffold integrity [23]. Therefore, the primary adhesion of the implanted scaffold without further fixation devices may help to eliminate this discrepancy and improve results of fixation of the scaffold which are implanted arthroscopically.

The present study examines for the first time different arthroscopic fixation techniques of cartilage implants of the hip joint in an experimental setup. It demonstrates that bioinspired structural alterations of scaffolds may support arthroscopic application significantly. Since the role of hip arthroscopy and consequently the number of surgeons who apply this technique have significantly increased in the recent years our study presents an important impulse towards easier procedures and subsequently broader clinical application [25].

Furthermore, a stickier scaffold may allow cartilage repair as a simple one step procedure when extended fixation techniques, for example, transosseous fixation or biodegradable pin fixation, are impossible due to tight anatomical conditions or operational circumstances like the hip joint.

The presented technique is a single-step procedure. In a clinical study, Fontana et al. showed that the arthroscopic autologous chondrocyte transplantation (ACT) is superior to arthroscopic debridement of hip chondral defects [26]. However, ACT requires a two-stage operation which represents a burden for the patient. Combining our technique with an improved cartilage implant with Mfx, MSCs are attracted to immigrate into the bioinspired PM-scaffold. Thereby, bioinspired PM-scaffold depicts a matrix for the condrogenic differentiation of the MSCs.

In an arthroscopic hip study, Mancini and Fontana compared the clinical outcome of the arthroscopic matrixinduced autologous chondrocyte implant (MACI) with the autologous matrix-induced chondrogenesis (AMIC) techniques which is matchable to our technique [27]. The authors showed that the AMIC technique as a single-stage procedure is a valid procedure for improvement in patients concerned by chondral defects.

\section{Conclusions}

This study focuses on the arthroscopic application of enhanced cartilage implants on femoral hip cartilage lesions. Although this essential aspect is difficult to objectify, the authors of this paper underline the improved implantation conditions by using the PGA-PM-scaffold and eagerly anticipate the experiences of other arthroscopic hip surgeons. PGA-PM-scaffolds demonstrate increased observed initial fixation strength in cadaver femoral head defects relative to PGA-scaffold, particularly when fibrin glue is used for fixation. Further investigations including biomechanical adhesion analysis and studies with a greater number of cases are necessary.

\section{Conflict of Interests}

Dr. Christian Kaps is consultant of BioTissue Technologies $\mathrm{GmbH}$.

\section{Authors' Contribution}

Dr. Matthias Lahner and Dr. Christian Duif contributed equally to this paper.

\section{Acknowledgments}

The authors thank Priv.-Doz. Dr. N. Lahner for correcting the paper. The authors thank Waldemar Pluschzyk for his excellent technical assistance. PGA-scaffolds were supplied free of charge through Dr. Christian Kaps of BioTissue Technologies $\mathrm{GmbH}$, Berlin, Germany. The study was supported by the Ministry for Innovation, Science and Research of the State of North Rhine-Westphalia, Germany, through the programme FH STRUCTUR 2014 to TS. 


\section{References}

[1] J. R. Steadman, W. G. Rodkey, K. K. Briggs, and J. J. Rodrigo, "The microfracture technique to treat full thickness articular cartilage defects of the knee joint," Orthopade, vol. 28, no. 1, pp. 26-32, 1999.

[2] W. G. Steadman Rodkey Jr. and J. J. Rodrigo, "Microfracture: surgical technique and rehabilitation to treat chondral defects," Clinical Orthopaedics and Related Research, supplement 391, pp. S362-S369, 2001.

[3] I. H. Beiser and I. O. Kanat, "Subchondral bone drilling: a treatment for cartilage defects," Journal of Foot Surgery, vol. 29, no. 6, pp. 595-601, 1990.

[4] S. A. Hunt, L. M. Jazrawi, and O. H. Sherman, "Arthroscopic management of osteoarthritis of the knee," The Journal of the American Academy of Orthopaedic Surgeons, vol. 10, no. 5, pp. 356-363, 2002.

[5] G. Kish, L. Módis, and L. Hangody, "Osteochondral mosaicplasty for the treatment of focal chondral and osteochondral lesions of the knee and talus in the athlete: rationale, indications, techniques, and results," Clinics in Sports Medicine, vol. 18, no. 1, pp. 45-66, 1999.

[6] M. Brittberg, A. Lindahl, A. Nilsson, C. Ohlsson, O. Isaksson, and L. Peterson, "Treatment of deep cartilage defects in the knee with autologous chondrocyte transplantation," The New England Journal of Medicine, vol. 331, no. 14, pp. 889-895, 1994.

[7] P. C. Kreuz, S. Müller, U. Freymann et al., "Repair of focal cartilage defects with scaffold-assisted autologous chondrocyte grafts: clinical and biomechanical results 48 months after transplantation," The American Journal of Sports Medicine, vol. 39, no. 8, pp. 1697-1705, 2011.

[8] S. Panseri, A. Russo, C. Cunha et al., "Osteochondral tissue engineering approaches for articular cartilage and subchondral bone regeneration," Knee Surgery, Sports Traumatology, Arthroscopy, vol. 20, no. 6, pp. 1182-1191, 2012.

[9] K. Mithoefer, R. J. Williams III, R. F. Warren et al., "The microfracture technique for the treatment of articular cartilage lesions in the knee: a prospective cohort study," Journal of Bone and Joint Surgery A, vol. 87, no. 9 I, pp. 1911-1920, 2005.

[10] P. Niemeyer, W. Koestler, and N. P. Südkamp, "Problems and complications of surgical techniques for treatment of fullthickness cartilage defects," Zeitschrift für Orthopädie und Unfallchirugie, vol. 149, no. 1, pp. 45-51, 2011.

[11] C. Erggelet, M. Endres, K. Neumann et al., "Formation of cartilage repair tissue in articular cartilage defects pretreated with microfracture and covered with cell-free polymer-based implants," Journal of Orthopaedic Research, vol. 27, no. 10, pp. 1353-1360, 2009.

[12] R. Dorotka, U. Windberger, K. Macfelda, U. Bindreiter, C. Toma, and S. Nehrer, "Repair of articular cartilage defects treated by microfracture and a three-dimensional collagen matrix," Biomaterials, vol. 26, no. 17, pp. 3617-3629, 2005.

[13] J. Kramer, F. Böhrnsen, U. Lindner, P. Behrens, P. Schlenke, and J. Rohwedel, "In vivo matrix-guided human mesenchymal stem cells," Cellular and Molecular Life Sciences, vol. 63, no. 5, pp. 616626, 2006.

[14] J. Gille, E. Schuseil, J. Wimmer, J. Gellissen, A. P. Schulz, and P. Behrens, "Mid-term results of Autologous Matrix-Induced Chondrogenesis for treatment of focal cartilage defects in the knee," Knee Surgery, Sports Traumatology, Arthroscopy, vol. 18, no. 11, pp. 1456-1464, 2010.
[15] J. P. Benthien and P. Behrens, "The treatment of chondral and osteochondral defects of the knee with autologous matrix-induced chondrogenesis (AMIC): method description and recent developments," Knee Surgery, Sports Traumatology, Arthroscopy, vol. 19, no. 8, pp. 1316-1319, 2011.

[16] T. Zantop and W. Petersen, "Arthroscopic implantation of a matrix to cover large chondral defect during microfracture," Arthroscopy: Journal of Arthroscopic and Related Surgery, vol. 25, no. 11, pp. 1354-1360, 2009.

[17] J. M. Patrascu, U. Freymann, C. Kaps, and D. V. Poenaru, "Repair of a post-traumatic cartilage defect with a cell-free polymer-based cartilage implant: a follow-up at two years by MRI and histological review," Journal of Bone and Joint Surgery $B$, vol. 92, no. 8, pp. 1160-1163, 2010.

[18] S. Knecht, C. Erggelet, M. Endres, M. Sittinger, C. Kaps, and E. Stüssi, "Mechanical testing of fixation techniques for scaffoldbased tissue-engineered grafts," Journal of Biomedical Materials Research B Applied Biomaterials, vol. 83, no. 1, pp. 50-57, 2007.

[19] S. Zelle, T. Zantop, S. Schanz, and W. Petersen, "Arthroscopic techniques for the fixation of a three-dimensional scaffold for autologous chondrocyte transplantation: structural properties in an in vitro model," Arthroscopy: The Journal of Arthroscopic \& Related Surgery, vol. 23, no. 10, pp. 1073-1078, 2007.

[20] M. Herbort, S. Zelle, D. Rosenbaum et al., "Arthroscopic fixation of matrix-associated autologous chondrocyte implantation: importance of fixation pin angle on joint compression forces," Arthroscopy, vol. 27, no. 6, pp. 809-816, 2011.

[21] M. Sittinger, C. Perka, O. Schultz, T. Häupl, and G.R. Burmester, "Joint cartilage regeneration by tissue engineering," Zeitschrift für Rheumatologie, vol. 58, no. 3, pp. 130-135, 1999.

[22] V. M. Ilizaliturri Jr., J. W. T. Byrd, T. G. Sampson et al., "A geographic zone method to describe intra-articular pathology in hip arthroscopy: cadaveric study and preliminary report," Arthroscopy, vol. 24, no. 5, pp. 534-539, 2008.

[23] J. E. J. Bekkers, A. I. Tsuchida, J. Malda et al., "Quality of scaffold fixation in a human cadaver knee model," Osteoarthritis and Cartilage, vol. 18, no. 2, pp. 266-272, 2010.

[24] R. J. Custers, W. J. Dhert, M. H. van Rijen, A. J. Verbout, L. B. Creemers, and D. B. Saris, "Articular damage caused by metal plugs in a rabbit model for treatment of localized cartilage defects," Osteoarthritis and Cartilage, vol. 15, no. 8, pp. 937-945, 2007.

[25] J. McCarthy and S. Mc Millan, "Arthroscopy of the hip: factors affecting outcome," Orthopedic Clinics of North America, vol. 44, pp. 489-498, 2013.

[26] A. Fontana, A. Bistolfi, M. Crova, F. Rosso, and G. Massazza, "Arthroscopic treatment of hip chondral defects: autologous chondrocyte transplantation versus simple debridement-a pilot study," The Arthroscopy-Journal of Arthroscopic and Related Surgery, vol. 28, no. 3, pp. 322-329, 2012.

[27] D. Mancini and A. Fontana, "Five-year results of arthroscopic techniques for the treatment of acetabular chondral lesions in femoroacetabular impingement," International Orthopaedics, 2014. 


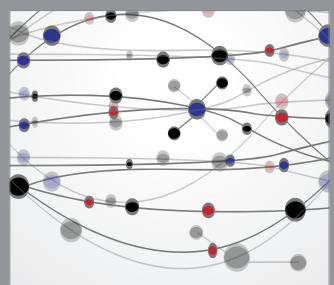

The Scientific World Journal
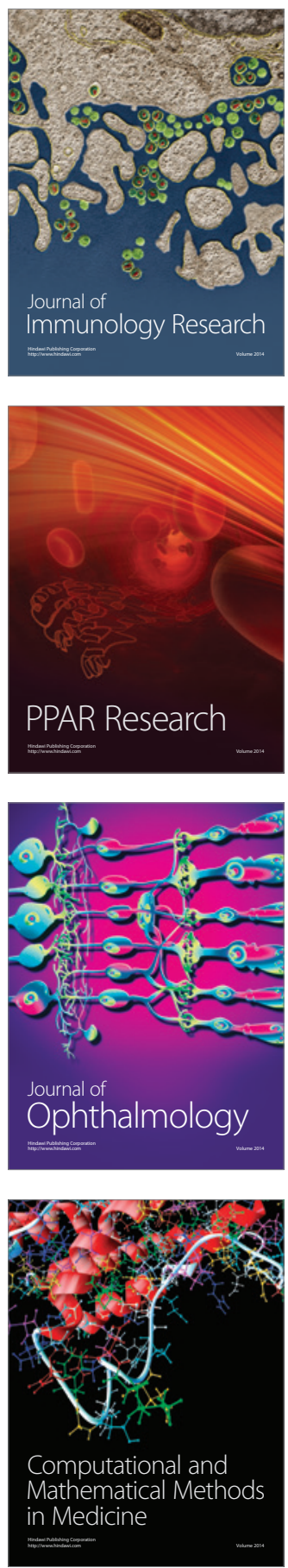

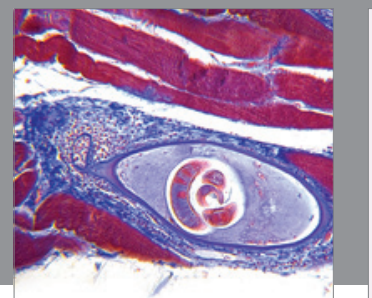

Gastroenterology

Research and Practice
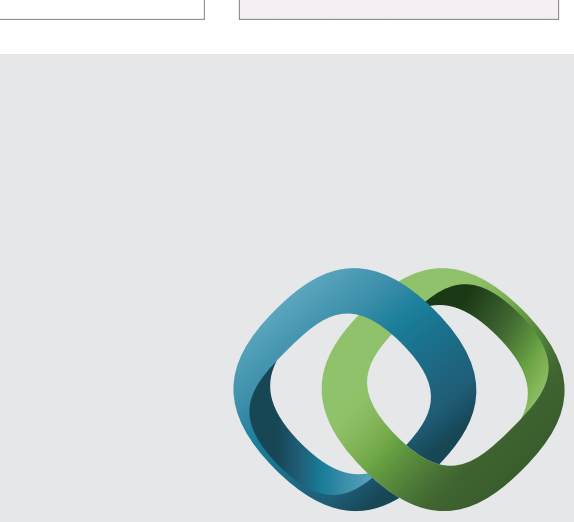

\section{Hindawi}

Submit your manuscripts at

http://www.hindawi.com
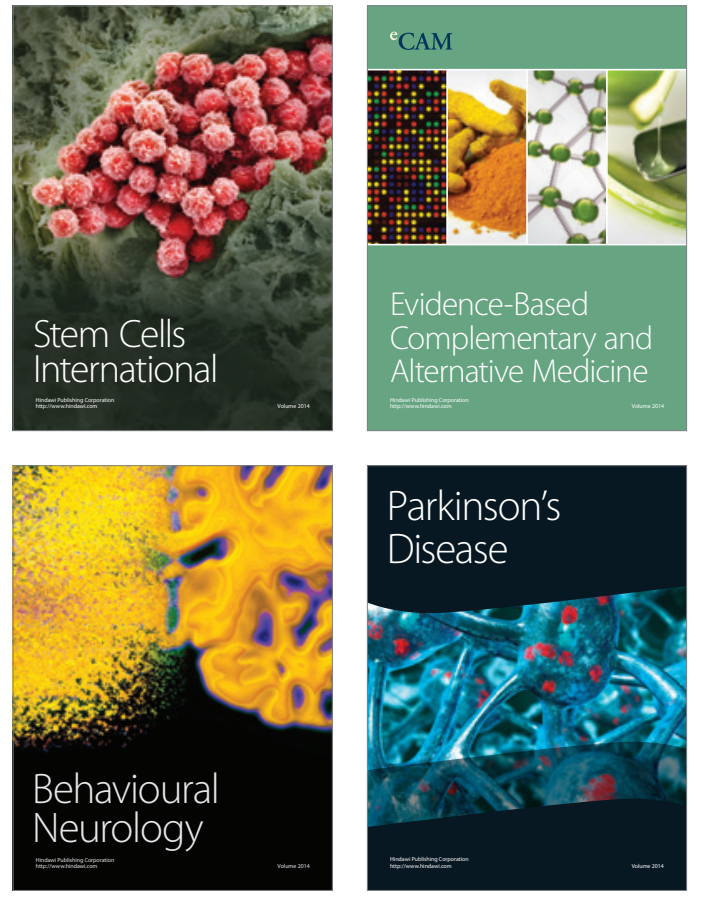
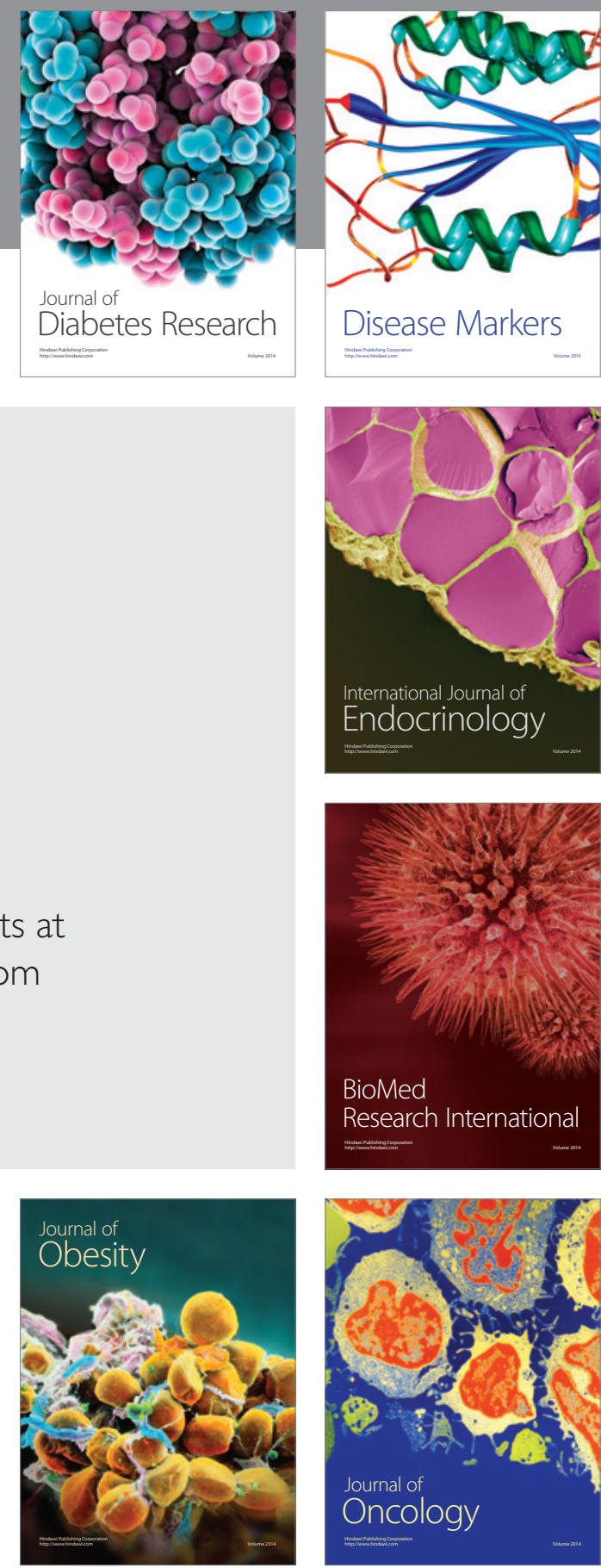

Disease Markers
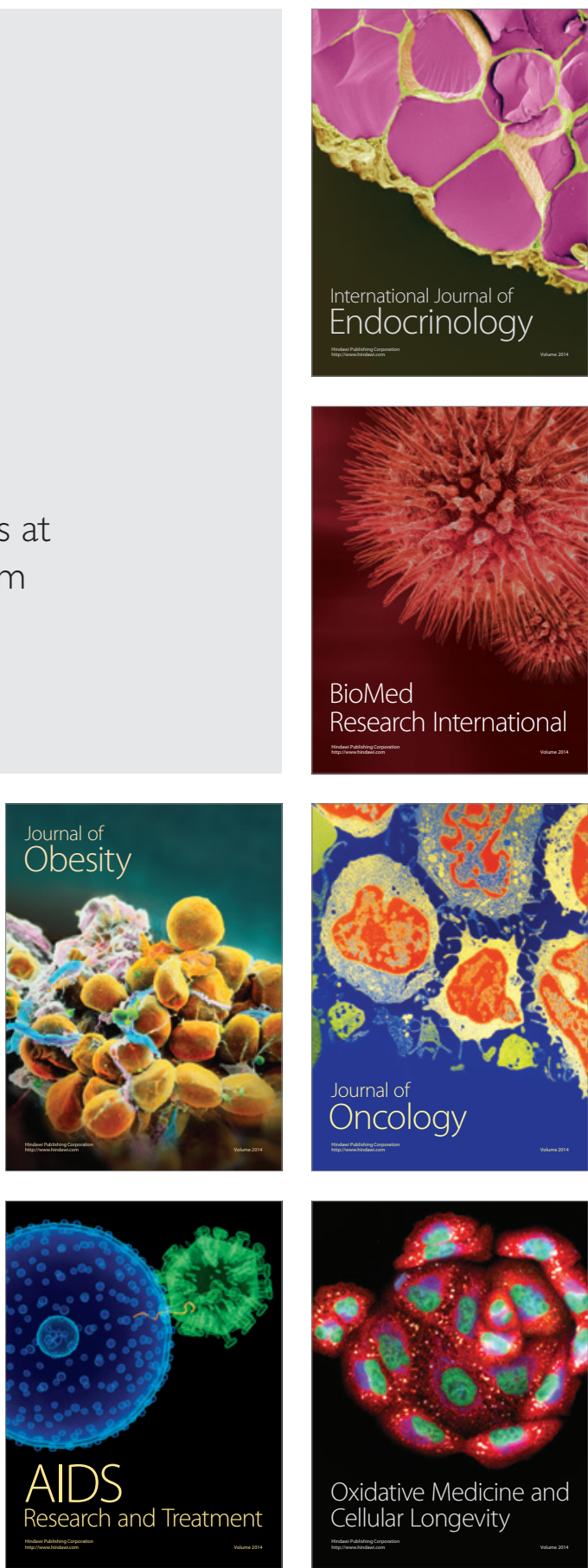